\title{
Determinantes ambientales que afectan el perfil de salud de población del Meta 2012-2014
}

\section{Environmental determinants that affect population health profile} of Meta 2012-2014

\section{Determinantes ambientais que afetam o perfil de saúde da população do Meta 2012-2014}

\author{
Óscar A. Gutiérrez-Lesmes ${ }^{1}$ \\ 1 Enfermero, MSc, Escuela de Salud Pública, Facultad de Ciencias de la Salud, Universidad de los Llanos \\ Email: oagutierrez@unillanos.edu.co
}

Recibido: 04 de marzo de 2016

Aceptado: 28 de julio de 2017

\begin{abstract}
Resumen
Se construyeron indicadores sintéticos basados en la morbilidad y la mortalidad como método para describir los factores ambientales presentes en los municipios del Meta en el periodo 2012 a 2014. Mediante transversal retrospectivo, utilizando las bases de datos estatales de vigilancia en salud pública y el registro único de afiliados (RUAF) de 2012 a 2014 , las cuales posterior a validación fueron analizadas en fases: construcción de los indicadores simples, índices temáticos e indicadores sintéticos. Se obtienen cuatro indicadores que permiten medir el grado de afectación en salud de cada uno de los municipios del Meta y la diferencia entre los mismos, permitiendo evidenciar la existencia de efectos diferenciales en cada municipio relacionados con factores ambientales como: sustancias químicas, agua, aire y fauna, la evidencia sobre la existencia de un efecto causado por estos factores se deriva de la presencia de morbilidad y mortalidad en diferentes eventos en salud.
\end{abstract}

Palabras Clave: Ambiente, Salud Pública, Salud Ambiental, Factores Epidemiológicos.

\begin{abstract}
Synthetic indicators based on morbidity and mortality were constructed as a method to describe the environmental factors present in the Meta municipalities in the period 2012 to 2014. Through a retrospective cross-section, using the public databases of surveillance in public health and the single registry of affiliates (RUAF) from 2012 to 2014, which after validation were analyzed in phases: construction of simple indicators, thematic indexes and synthetic indicators. Four indicators are obtained that allow measuring the degree of health impairment of each of the municipalities of the Meta and the difference between them, allowing evidence of the existence of differential effects in each municipality related to environmental factors such as: chemical substances, water, air and fauna, the evidence on the existence of an effect caused by these factors is derived from the presence of morbidity and mortality in different health events.
\end{abstract}

Key words: Environment, Public Health, Environmental Health, Epidemiological Factors. 


\begin{abstract}
Resumo
Foram construidos indicadores sintéticos baseados na morbilidade e mortalidade como método para descrever os fatores ambientais presentes nos municípios do Meta no período de 2012 a 2014 . Através de uma retrospectiva transversal, utilizando as bases de dados estatísticos de vigilância na saúde pública e de registro único de filiados (RUAF) de 2012 a 2014 , as quais após validação foram analisadas em fases: construção de indicadores simples, índices temáticos e indicadores sintéticos. Se obtêm quatro indicadores que permitem medir o grau de afetividade em cada um dos municípios do Meta e a diferença entre os mesmos, permitindo evidenciar a existência de efeitos diferenciais em cada município relacionados com fatores ambientais como: substâncias químicas, água, ar e fauna, a evidência sobre a existência de um efeito por estes fatores deriva-se da presencia de morbilidade e mortalidade em diferentes eventos em saúde.
\end{abstract}

Palavras Chave: Ambiente, Saúde Pública, Saúde Ambiental, Fatores Epidemiológicos.

\section{Introducción}

Los indicadores generados en esta investigación obedecen a una metodología transversal basada en los impactos ya ocurridos en la población, con el objetivo de describir el impacto de factores ambientales sobre las salud de los habitantes delos municipios del Meta, los resultados reflejan un comportamiento específico en los municipios del departamento del Meta, caracterizando cada uno de estos según el tipo de evento de morbilidad y mortalidad, estos eventos son considerados por la OMS como efecto de diversos factores ambientales (Ordóñez, 2000; Posada, Carroquino, y Soldevilla, 2004; Yassi, Kjellstrom, Dekok, y Guidotti, 2002).

El modelo de determinantes sociales reconoce que los principales determinantes de la salud proceden mayoritariamente del ambiente (Adolfo Gerardo, García Fariñas, y Bonet Gorbea, 2007; Villar, 2007). El nivel de salud de la población está condicionado por el ambiente representado en factores: biológicos, estrato social, saneamiento básico, el acceso a servicios, el empleo y la vivienda (Moiso y Barragán, 2007; Pérez Jiménez, Diago Garrido, Corona Miranda, Espinosa Díaz, y González Pérez, 2011).

El Ministerio de Salud y Protección Social en el documento dimensiones prioritarias en salud pública del Plan Decenal de Salud Pública 2012-2021 estima que: "el $41 \%$ de los colombianos viven en grandes ciudades en donde se encuentra la mayor concentración de contaminantes en el aire como $\mathrm{PM}_{10}, \mathrm{PM}_{2}, 5, \mathrm{CO}, \mathrm{NO}_{2}, \mathrm{SO}_{2}$ y $\mathrm{O}_{3}$, y además podrían darse otros tipos de contaminantes tales como el ruido, campos electromagnéticos y olores ofensi$\operatorname{vos}^{\prime \prime}(2012$, p. 23)

El ambiente genera el $24 \%$ de la morbilidad y el $25 \%$ de las muertes tempranas en el mundo, en los países en desarrollo el porcentaje de mortalidad atribuible a causas ambientales es del 25\%, y en desarrollados del 17\% (Prüss-Üstün y Annette Corvalán, 2006) y en me- nores de 15 años se atribuye el 36\% de la mortalidad a causas ambientales, esto es alrededor de la cuarta parte de la carga mundial de morbilidad. En América Latina se estima que más del $10 \%$ de todos los años de vida perdidos por discapacidad se deben directamente a factores ambientales, correspondiéndole al abastecimiento de agua y saneamiento básico el 5,5\%, y el 4,5\% restante a contaminación atmosférica urbana, sustancias químicas, desechos agroindustriales y contaminación del aire interior (Organización-Panamericana-de-la-Salud, 2001).

La interacción de los factores ambientales naturales y antrópicos con la población humana genera un comportamiento epidemiológico específico, en esta interacción cada factor ejerce un rol en la generación de causalidad para la presencia de morbimortalidad en una población. Según Ordoñez se han descrito más de 64 determinantes ambientales que participan en la morbimortalidad conocida en el mundo (Ordóñez, 2000), estos factores han sido clasificados en dos clases: naturales y antrópicos (Arturo, 2008); por otro lado, la Organización Mundial de la Salud ha descrito 85 enfermedades con su respectiva mortalidad, las cuales califica como ocasionadas por determinantes ambientales (Prüss-Üstün y Annette Corvalán, 2006), varias de estas enfermedades hacen parte del perfil epidemiológico del Meta.

\section{Metodología}

Se realizó un estudio transversal retrospectivo, los datos se obtuvieron de las bases de datos estatales de vigilancia en salud pública (SIVIGILA) y el registro único de afiliados (RUAF), de los años 2012, 2013, 2014 del departamento del Meta.

Las bases fueron filtradas mediante los siguientes criterios: la enfermedad o la causa de muerte estén clasificadas por la Organización Mundial de la Salud como morbimortalidad de causa ambiental (Quezada, 2006; World Health Organization, 2004) y el lugar de resi- 
dencia de la persona seleccionada por el tipo de evento fuese un municipio del departamento del Meta. Los demás casos fueron retirados de la base de datos bajo los siguientes criterios de exclusión: la enfermedad o la causa de muerte no están clasificadas por la Organización Mundial de la Salud como morbimortalidad de causa ambiental y/o el municipio de residencia y/o procedencia donde ocurre la morbimortalidad no es del departamento del Meta.

Una vez fueron seleccionados los casos de análisis se validaron y construyeron (datos repetidos, ajuste de caso, y lugar de residencia) y construyeron nuevas bases de datos, las cuales fueron analizadas en 3 fases: construcción de los indicadores simples, índices temáticos, los indicadores sintéticos.

En la Fase 1, para cada municipio se construyó la incidencia acumulada y la tasa de mortalidad por evento ocurrida en el periodo 2012-2014; los eventos a los cuales se les estimó la incidencia acumulada y tasa la mortalidad fueron: Intoxicación por Metales, Intoxicación por Plaguicidas, Intoxicación por Solventes, Intoxicación por Otras Sustancias Químicas, Accidente Ofídico, Agresiones Por Animales Potencialmente Transmisores de Rabia, Leptospirosis, Enfermedad Diarreica Aguda Global, Dengue Global, Paludismo en todas sus formas, Leishmaniasis, Enfermedad De Chagas, Fiebre Amarilla, ESI (enfermedad similar a influenza), IRA (infección respiratoria aguda), IRAG (infección respiratoria aguda grave), IRAG inusitado.

En la construcción de la incidencia y de la tasa de mortalidad se utilizó como denominador la media de la población de cada municipio para el período según reporte del Departamento Nacional de Estadística. Todas las tasas fueron calculadas usando una constante de 100.000 habitantes.

Para el cálculo de la incidencia acumulada (IA) de cada uno de los eventos se utilizó la siguiente fórmula:

$$
I A(\alpha *)=\frac{X^{*}\left(a^{\dagger}\right),\left(p^{\ddagger}\right),\left(e^{\S}\right)}{N(p),(e)} * K
$$

Para el cálculo de la tasa de mortalidad (TM) de cada uno de los eventos se utilizó la siguiente fórmula:

$$
T M(a *)=\frac{X^{* *}\left(a^{++}\right)(p),(e)}{N^{++}} * K
$$

* Número de total de casos de morbilidad

$\dagger \quad$ Representa la morbilidad para la cual se calcula la incidencia.

‡ Periodo de ocurrencia del evento

$\S \quad$ Especio o lugar donde ocurre el evento
** Número total de casos de mortalidad

†† Representa la causa de muerte para la cual se calcula la tasa.

㧊 Población a mitad de periodo y lugar

En la fase 2 se construyeron índices temáticos no ponderados con las incidencias y con las tasas de mortalidad de la primera fase, para la construcción de los índices temáticos no ponderados se utilizaron las siguientes formulas:

$$
\begin{aligned}
& \text { 1. Índice temático }(I T I A)=\sum_{i=1}^{n} I A\left(r t a^{S S}\right) \\
& \text { 2. Índice temático } \quad(I T T M)=\sum_{i=1}^{n} I A\left(r t m^{* * *}\right)
\end{aligned}
$$

$\S \S \quad$ rta, corresponde a un indicador simple, el cual tiene relación temática con el índice temático que se cons truye.

*** rtm, corresponde a un indicador simple, el cual tiene relación temática con el índice temático que se cons truye.

Cada índice temático es la agrupación de las incidencias o de tasas de mortalidad, basados en la clasificación por grandes causas y su historia natural, de esta manera se construyeron las siguientes agrupaciones: intoxicaciones químicas, zoonosis, infección gastrointestinal, enfermedades transmitidas por vectores e infecciones respiratorias.

En la fase final (fase 3) Se construyeron indicadores sintético por municipio, en el marco teórico de indicadores de desarrollo sostenible del Decreto 1200 de 2004 del Ministerio de Ambiente, Vivienda y Desarrollo (Ministerio de Ambiente, 2004) los cuales reconocen el impacto del ambiente derivado de la calidad del agua y la calidad del aire; estos indicadores buscan evidenciar el impacto de la gestión ambiental orientada hacia el desarrollo sostenible (Schuschny y Soto, 2009). Y en la metodología DPSEEA, la cual determina: (Fuerzas impulsoras $\rightarrow$ Presión $\rightarrow$ Estado $\rightarrow$ Exposición $\rightarrow$ Efecto, Acción) (Posada, Carronquino, y Soldevilla, 2004).

Para la construcción de los indicadores sintéticos se utilizó la siguiente formula:

$$
\text { Indicador sintético } \sum_{i=1}^{n}\left(I T I A^{t+t}+I T T M^{\ddagger \neq \neq}\right)
$$

††† ITIA corresponde a un índice temático de incidencia acumulada clasificado en un factor

㧊 ITTM corresponde a un índice temático de mortali dad clasificado en un factor ambiental

Estos indicadores no son medición del estado ambiental, pero si representan la existencia de un efecto procedente de un estado ambiental, estos indicadores de muestran la existencia de factores ambientales presentes afectando el perfil de salud de los habitantes del Meta. 
En la sumatoria de los casos de morbilidad y mortalidad dejaron de ser considerados casos para asumir el valor de puntos representados en una escala, los efectos sobre la salud fueron agrupados en cuatro grupos similares a los determinados por la OMS en la metodología DPSEEA, obteniendo como resultado los cuatro indicadores. Una vez determinados los indicadores se clasificaron los municipios en tres categorías (baja, media y alta) en cada uno de los indicadores sintéticos, la clasificación se realizó mediante los percentiles para la elaboración de mapas temáticos.

\section{Resultados}

La clasificación de los municipios según cuantificación de los indicadores evidencia marcadas diferencias de carga ambiental entre los municipios, diferenciados según factores ambiental relacionado con las morbilidad y mortalidad ocurrida, en las siguientes tablas de puede evidenciar el comportamiento por municipio y en los mapas se observa la conformación de zonas donde confluyen comportamientos para factores ambientales.

En la tabla 1 se evidencia los municipios en orden descendente según el puntaje obtenido en el indicador sintético para el factor ambiental calidad, acceso y manejo del recurso hídrico, evidenciando el grado de afectación de los municipios según el impacto en salud relacionada con el recurso hídrico.

En el Mapa 1, se observan los estratos construidos para el indicador sintético para el factor ambiental calidad, acceso y manejo del recurso hídrico. Los estratos fueron definidos mediante percentiles y los municipios se clasificaron según su ubicación en alta, media y baja exposición. Obsérvese la conformación de zonas geográfica que agrupan municipios.

En la tabla 2 se observan los municipios en orden descendente según el puntaje obtenido en el indicador sintético para el factor ambiental en la calidad del aire, evidenciando el grado de afectación de los municipios según el impacto en salud.

En el Mapa 2, se observan los estratos construidos para el indicador sintético factor ambiental en la calidad del aire. Los estratos fueron definidos mediante percentiles y los municipios se clasificaron según su ubicación en alta, media y baja necesidad

\section{Indicador sintético para el factor ambiental denominado Fauna}

La tabla 3 evidencia los municipios en orden descendente según el puntaje obtenido en el indicador sinté-
Tabla 1. Indicador sintético para el factor ambiental denominado calidad acceso y manejo del recurso hídrico.

\begin{tabular}{|c|c|}
\hline Municipio & Puntaje \\
\hline El Castillo & 7920,77 \\
\hline San Juan de Arama & 7177,52 \\
\hline El Calvario & 6070,90 \\
\hline Restrepo & 4727,25 \\
\hline Cabuyaro & 4169,95 \\
\hline Vistahermosa & 3755,49 \\
\hline El Dorado & 3608,80 \\
\hline La Macarena & 3415,45 \\
\hline La Uribe & 3318,19 \\
\hline Cubarral & 3285,99 \\
\hline Puerto López & 3073,52 \\
\hline San Carlos de Guaroa & 2827,18 \\
\hline Barranca de Upía & 2582,78 \\
\hline Puerto Rico & 2568,23 \\
\hline Guamal & 2312,53 \\
\hline Mapiripán & 2209,56 \\
\hline Acacias & 1847,71 \\
\hline Fuentedeoro & 1643,23 \\
\hline Puerto Gaitán & 1613,32 \\
\hline Lejanías & 1273,84 \\
\hline San Martín & 1238,74 \\
\hline Puerto Concordia & 1044,62 \\
\hline San Juanito & 936,40 \\
\hline Mesetas & 729,82 \\
\hline Puerto Lleras & 678,91 \\
\hline Villavicencio & 643,44 \\
\hline Granada & 603,34 \\
\hline Cumaral & 431,02 \\
\hline Castilla la Nueva & 409,98 \\
\hline
\end{tabular}

tico para el factor ambiental Faunístico, evidenciando el grado de afectación de los municipios según el impacto en salud relacionado con la fauna.

En el Mapa 3, se observan los estratos construidos para el indicador sintético para el factor ambiental de la fauna. Los estratos fueron definidos mediante percentiles y los municipios se clasificaron según su ubicación en alta, media y baja necesidad. 
Tabla 2. Indicador sintético para el factor ambiental denominado calidad del aire.

\begin{tabular}{|c|c|}
\hline Municipio & Puntaje \\
\hline El Calvario & 5649,93 \\
\hline Puerto Gaitán & 5210,76 \\
\hline El Dorado & 4400,98 \\
\hline San Juan de Arama & 3412,45 \\
\hline Guamal & 3119,05 \\
\hline Cabuyaro & 2964,78 \\
\hline Restrepo & 2952,57 \\
\hline San Martín & 2641,56 \\
\hline Cumaral & 2348,24 \\
\hline Puerto López & 2216,01 \\
\hline Cubarral & 2069,92 \\
\hline Barranca de Upía & 2026,49 \\
\hline Granada & 1550,85 \\
\hline San Carlos de Guaroa & 1467,63 \\
\hline Castilla la Nueva & 1275,07 \\
\hline El Castillo & 1275,05 \\
\hline Villavicencio & 1241,33 \\
\hline La Uribe & 1207,82 \\
\hline Fuentedeoro & 1181,56 \\
\hline Lejanías & 970,05 \\
\hline Puerto Rico & 924,56 \\
\hline Mapiripán & 903,38 \\
\hline Acacías & 849,40 \\
\hline Puerto Concordia & 834,48 \\
\hline San Juanito & 766,96 \\
\hline Mesetas & 701,97 \\
\hline La Macarena & 560,93 \\
\hline Puerto Lleras & 535,15 \\
\hline Vistahermosa & 414,79 \\
\hline
\end{tabular}

\section{Indicador sintético para el factor ambiental denominado Sustancias Químicas}

La tabla 4. Evidencia los municipios en orden descendente según el puntaje obtenido en el indicador sintético para el factor ambiental de sustancias químicas, evidenciando el grado de afectación de los municipios según el impacto en salud relacionado con sustancias.
Tabla 3. Indicador sintético para el factor ambiental denominado Fauna.

\begin{tabular}{|l|l|}
\hline \multicolumn{1}{|c|}{ Municipio } & Puntaje \\
\hline Vistahermosa & 2139,02 \\
\hline Restrepo & 1978,85 \\
\hline Puerto Rico & 1894,78 \\
\hline San Juan de Arama & 1842,72 \\
\hline El Dorado & 1701,71 \\
\hline Castilla la Nueva & 1679,53 \\
\hline San Carlos de Guaroa & 1513,14 \\
\hline Guamal & 1456,00 \\
\hline Puerto Gaitán & 1403,53 \\
\hline Puerto López & 1374,58 \\
\hline Cabuyaro & 1293,86 \\
\hline Acacias & 1270,30 \\
\hline Barranca de Upía & 1245,03 \\
\hline San Martín & 1186,79 \\
\hline Cubarral & 1138,45 \\
\hline Villavicencio & 1120,29 \\
\hline Cumaral & 1033,56 \\
\hline Fuentedeoro & 1007,60 \\
\hline El castillo & 865,49 \\
\hline Mapiripán & 821,25 \\
\hline La Uribe & 743,28 \\
\hline Lejanías & 549,81 \\
\hline Granada & 502,06 \\
\hline Puerto Concordia & 466,90 \\
\hline La Macarena & 404,49 \\
\hline Mesetas & 375,91 \\
\hline Puerto Lleras & \\
\hline El Calvario & \\
\hline San Juanito & 179 \\
\hline
\end{tabular}

En el Mapa 4, se observan los estratos construidos para el indicador sintético para el factor ambiental de sustancias químicas. Los estratos fueron definidos mediante percentiles y los municipios se clasificaron según su ubicación en alta, media y baja necesidad.

\section{Discusión}

De los impactos en salud (perfiles de morbilidad y mortalidad) encontrados en esta investigación, se sugiere la presencia de efectos provenientes de características propias de: el agua (Córdoba, Del Coco, y 


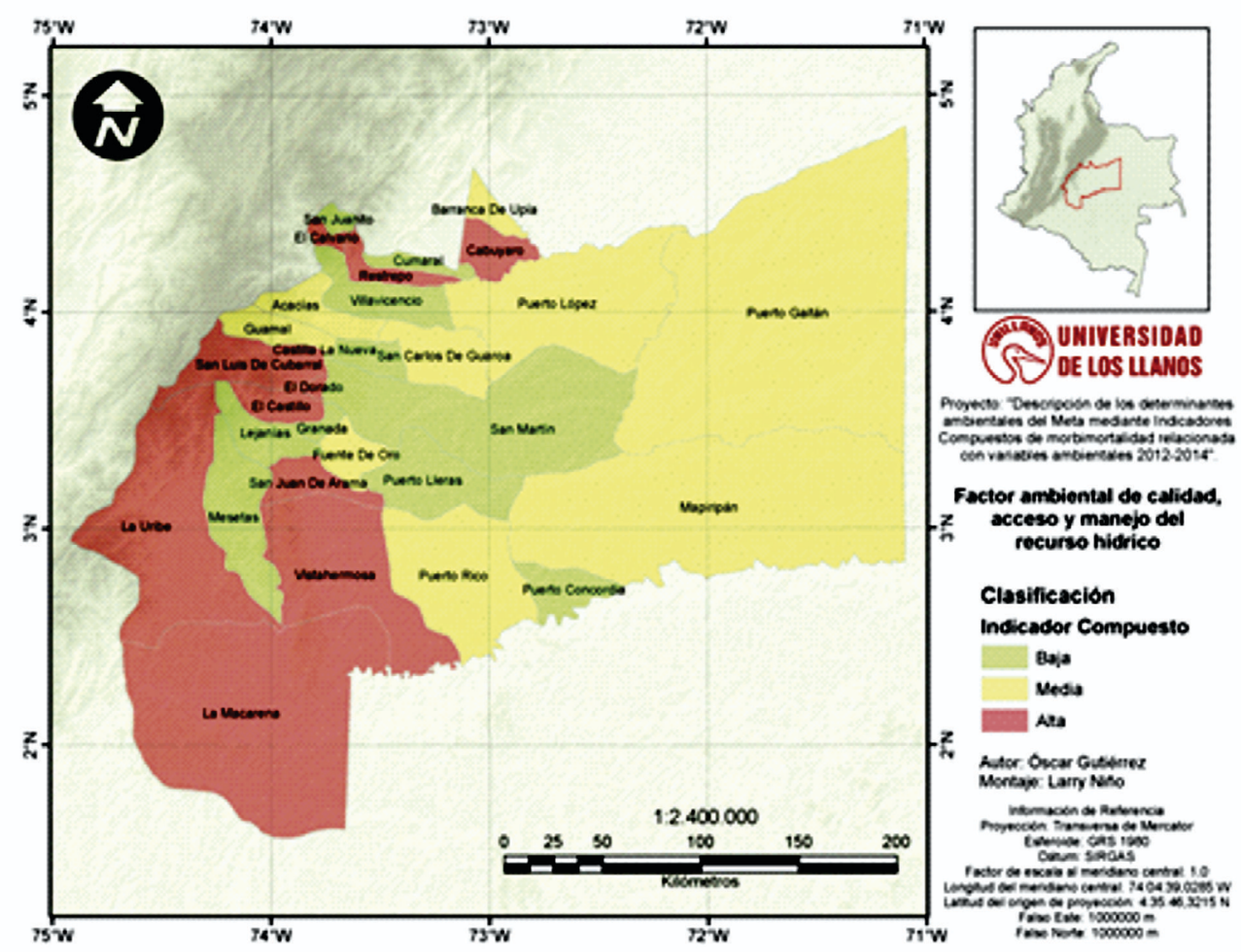

Mapa 1. Factor ambiental de la calidad acceso y manejo del recurso hídrico

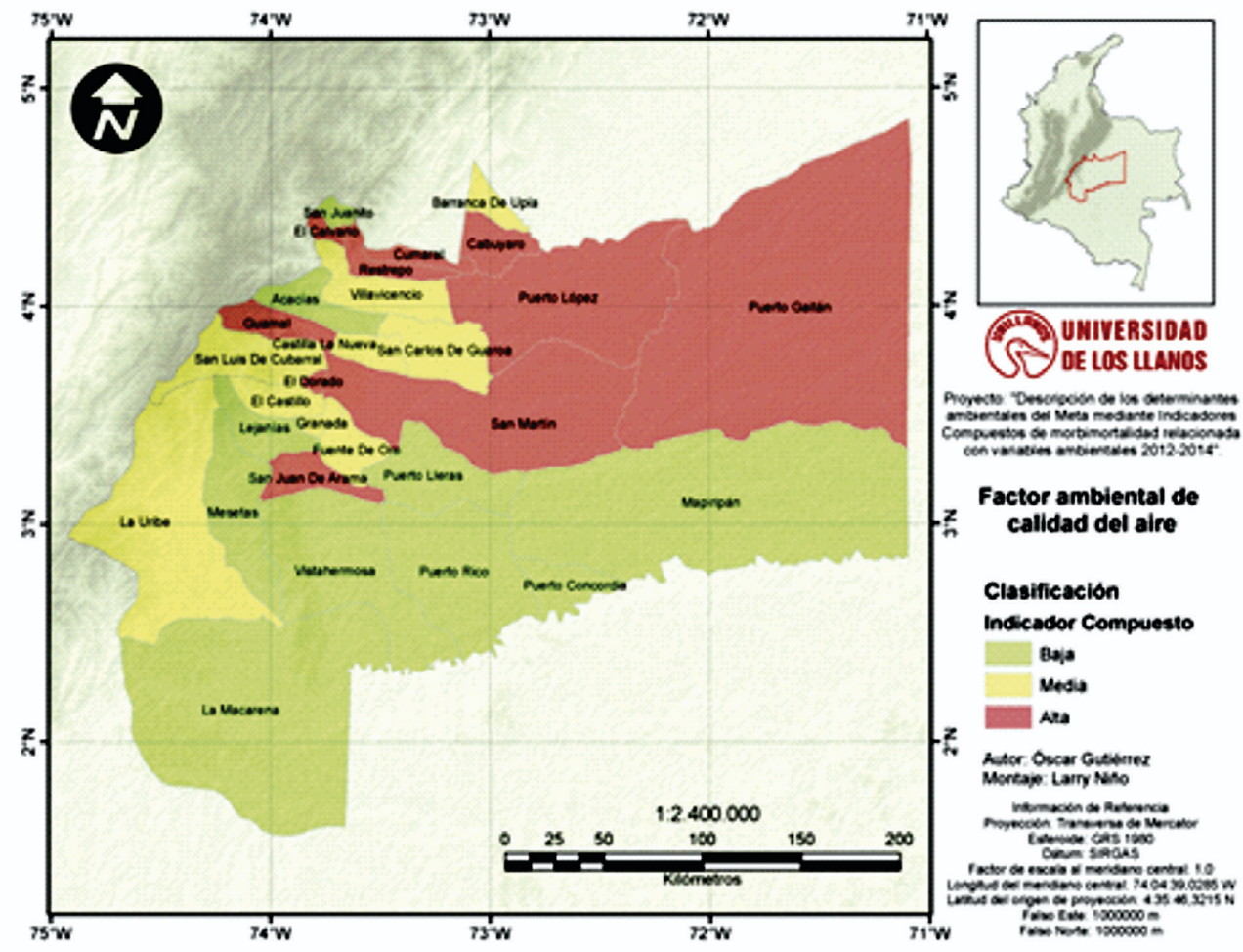

Mapa 2. Factor ambiental denominado calidad del aire 


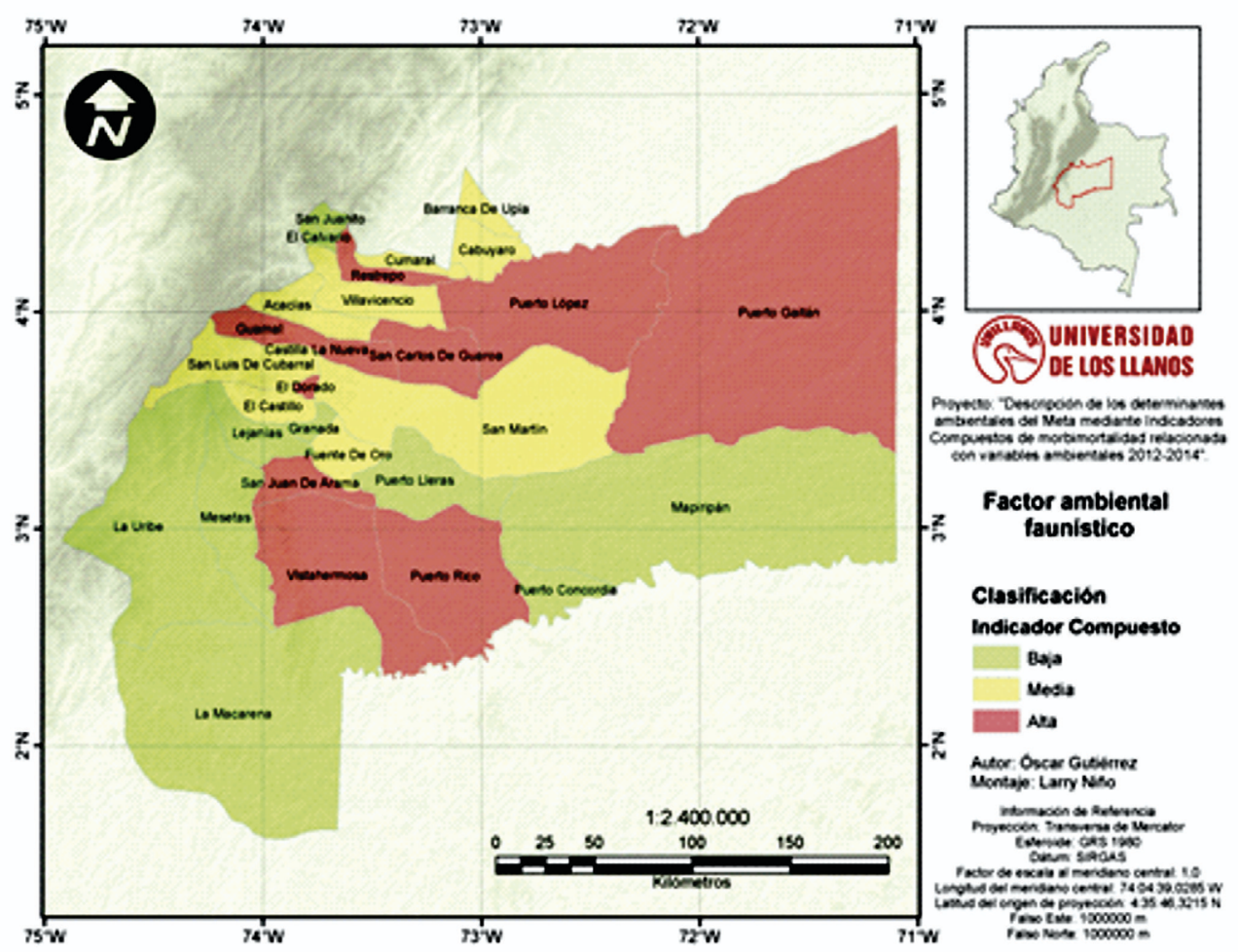

Mapa 3. Factor ambiental Faunístico.

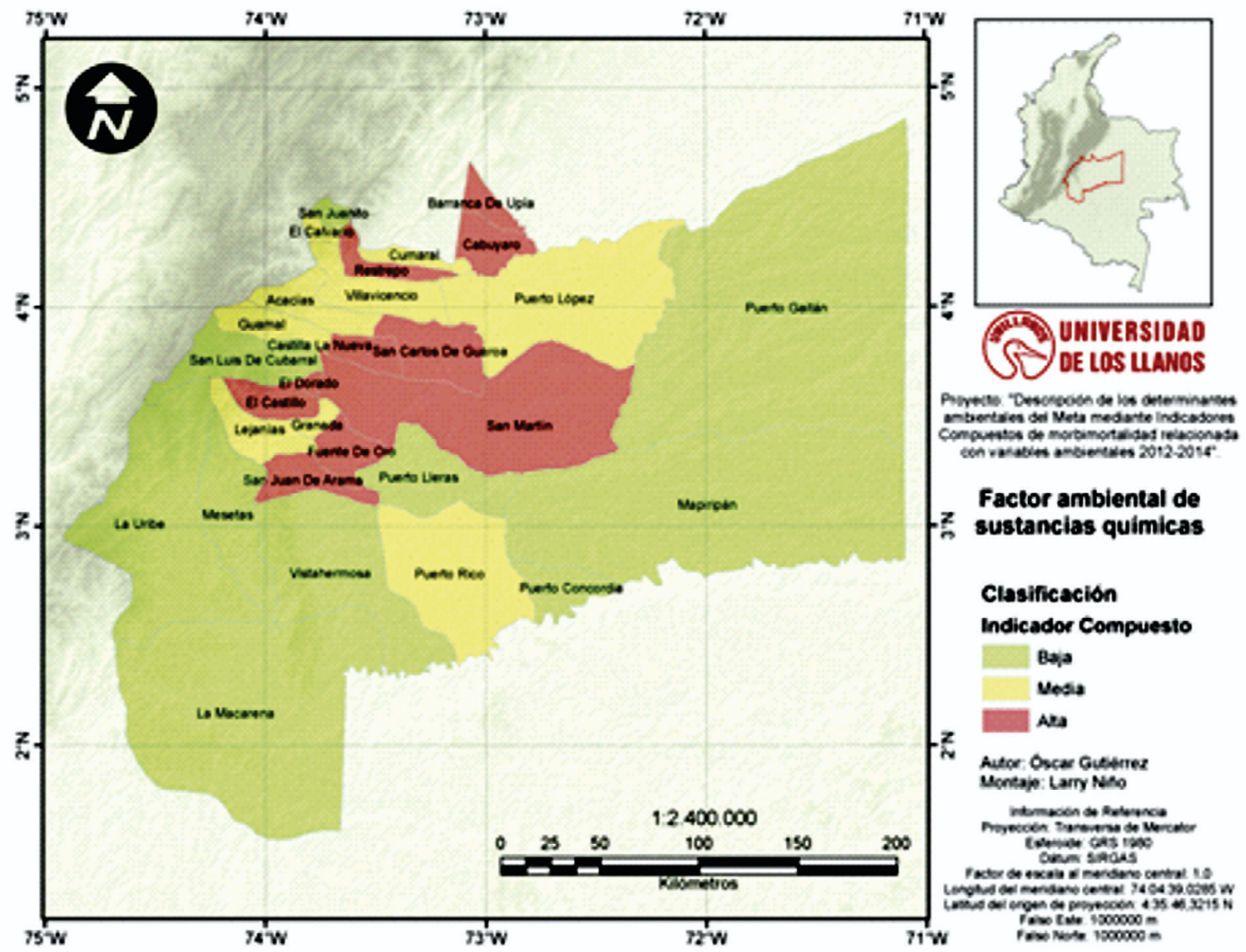

Mapa 4. Factor ambiental sustancias químicas 
Tabla 4. Indicador sintético para el factor ambiental denominado Sustancias Químicas.

\begin{tabular}{|c|c|}
\hline Municipio & Puntaje \\
\hline Fuentedeoro & 890,62 \\
\hline San Juan de Arama & 750,74 \\
\hline San Carlos de Guaroa & 719,79 \\
\hline El castillo & 574,88 \\
\hline San Martín & 542,96 \\
\hline Cabuyaro & 506,80 \\
\hline Barranca de Upía & 503,31 \\
\hline Castilla la Nueva & 430,54 \\
\hline Restrepo & 418,80 \\
\hline El Dorado & 410,76 \\
\hline El Calvario & 354,51 \\
\hline Puerto López & 327,40 \\
\hline Cumaral & 309,72 \\
\hline Granada & 304,37 \\
\hline Guamal & 280,12 \\
\hline Acacias & 243,12 \\
\hline Villavicencio & 229,36 \\
\hline Lejanías & 217,91 \\
\hline Puerto Rico & 197,85 \\
\hline Cubarral & 189,74 \\
\hline Puerto Gaitán & 182,28 \\
\hline Puerto Lleras & 148,19 \\
\hline La Uribe & 119,59 \\
\hline Mesetas & 90,79 \\
\hline Vistahermosa & 78,78 \\
\hline La Macarena & 70,92 \\
\hline Mapiripán & 31,29 \\
\hline San Juanito & 5,95 \\
\hline Puerto Concordia & 1,52 \\
\hline
\end{tabular}

Basualdo, 2010; Oller-Arlandis y Sanz-Valero, 2012; Tobón y López, 2011), el aire (De La Rosa, Mosso, y Ullán, 2002; Gavidia, Pronczuk, y Sly, 2009; Melgar Pérez, 2013), las sustancia químicas (Antonio, Hasen, y M, 2011; Valderrama, Baena, y Pérez, 2012) y la fauna (Cruz-Reyes, 2009; Ramos, 2010), la diferencia en las tasas de mortalidad y mortalidad entre los municipios evidencia que cada factor se expresa de manera específica cada municipio. En los siguientes párrafos se discutirán factores ambientales mencionados anteriormente, y su comportamiento como determinantes de efectos ambientales sobre la salud.

El agua es un determinante en la salud: como sustento básico para la salud, resguarda las condiciones sanitarias de las poblaciones (de Jesús Alba et al., 2013), un acceso inadecuado por déficit o agua con contaminación biológica o química, junto con el manejo inadecuado del agua en la vivienda, son factores determinantes en el proceso salud-enfermedad, la disponibilidad inmediata de agua potable hace posible un medio ambiente salubre que limita la propagación de enfermedades del hombre y de los animales (Córdoba et al., 2010). Las actividades humanas hacen manejo inadecuado de los desechos orgánicos y/o químicos los cuales terminan contaminando las fuentes hídricas naturales (Correa Restrepo, de la Ossa Arteaga, y VaIlejo Chanci, 2011).

La cobertura inadecuada de los acueductos aumenta el riesgo de enfermedades relacionadas con el agua debido a que las poblaciones deben extraerla de pozos profundos, obtenerla de ríos, fuentes la cuales no son aptas, ya que su calidad está afectada por contaminación con sustancias químicas (Antonio et al., 2011; Calleros et al., 2012; Oller-Arlandis y Sanz-Valero, 2012; Tobón y López, 2011; Valderrama et al., 2012) o biológicas (Espinoza, Segura, y Rodríguez, 2011; Esquicha, Falcón, y Oshiro, 2012; Guillen et al., 2013; Junkin y Eugene, 1988; Valenzuela, Godoy, Almonacid, y Barrientos, 2012), según la Organización Panamericana de la Salud este tipo de fuente contiene microorganismos autóctonos del medio y microorganismos patógenos (Silva, Villalobos, y Ortega, 2012), estos patógenos provienen de actividades antrópicas como el vertimiento de aguas servidas, procesos industriales, y actividades agropecuarias (Janet, Fernanda, y Parra, 2013) etc.

El fenómeno se hace aún más complejo ya que contar con acceso a agua potable por medio de acueductos no exime de la afectación de la salud causada por el consumo de agua (Álvarez Miño, Cantillo Matos, Rico Gutiérrez, y Salazar, 2013), en países donde existe un sistema de vigilancia de la calidad del agua ligado a la vigilancia epidemiológica, se ha determinado que parte de las enfermedades de origen en el agua se deben a fallas en el tratamiento del agua como la incorporación de agua contaminada en los acueductos o problemas de recrecimiento bacteriano en el sistema que distribuye el agua (Córdoba et al., 2010; Espinoza et al., 2011). 
Otra causa probable para que la disponibilidad de un buen sistema de abastecimiento de agua potable no sea suficiente para garantizar la no aparición de efectos sobre la salud, es el manejo inadecuado de recurso hídrico intradomiciliario, una correcta higiene sanitaria evita la contaminación del agua dispuesta en el interior de las viviendas (Jacinto, Aponte, y ArrunáteguiCorrea, 2012). Cuando el acceso es inadecuado se recurre al almacenamiento de agua en las viviendas convirtiendo a las viviendas en hábitat para vectores, en sitio reproducción del Aedes Aegypti (Ministerio de la Protección Social, 2012), vector transmisor del Dengue, sumado a esto la búsqueda cuerpos de agua para mantener el acceso al recurso expone a las poblaciones al Anofeles Darlingi (Escobedo, Hoyos, y Zavalaga, 2012; Medina et al., 2011) vector transmisor de la Malaria.

Las enfermedades gastrointestinales y las enfermedades transmitidas por vectores como Dengue y Malaria en todos los municipios del departamento del Meta, evidencian que el factor ambiental agua (acceso, calidad y el manejo del agua) está comportándose como un factor de riesgo ocasionando impactos sobre la salud. Los resultados del indicador sintético obtenidos arrojaron que existe diferencias en el impacto sobre los perfiles de salud en cada uno de los municipios, por ejemplo: El Castillo registra 7920 puntos en el indicador comparado con Castilla la Nueva con 409 puntos, lo anterior evidencia diferencias en los gradientes de exposición frente a las variables ligadas a la calidad, acceso y al manejo adecuado del agua en cada uno de los municipios.

El aire es un factor de riesgo cuando se encuentra contaminado o cuenta con características climáticas (temperatura y humedad) específicas (Aguinaga y Lazo, 2003), es importante mencionar que la calidad del aire puede estar afectada en exteriores o en interiores donde se produce la exposición que causa efectos sobre la salud (Finkelman, Corey, y Calderón, 1994; Gavidia et al., 2009; Rivas R, Barrios C, Dorner P, y Osorio S, 2008; Vieira et al., 2012).

La presencia de morbilidad y mortalidad en la poblaciones ligadas al sistema respiratorio y al sistema cardiovascular son evidencia de las condiciones atmosféricas a las que se exponen las poblaciones (Álvarez Sintes, Álvarez Sintes, Álvarez Castro, y Toledo Vila, 1997; Barrios Casas, Peña-Cortés, y Osses Bustingorry, 2004; Hernández-Flórez et al., 2013; Molina Esquivel y Meneses Ruiz, 2003; Prieto C, Mancilla F, Astudillo O, Reyes P, y Román A, 2007; Romero-Placeres et al., 2004). La incorporación del oxígeno para el metabolismo se realiza mediante la respiración pulmonar sien- do esta la vía por la cual ingresan sustancias químicas al sistema cardiovascular, por eso la mayor parte de las enfermedades originadas por las características del aire sean sobre el sistema respiratorio y el sistema cardiovascular (Castro et al., 2010; Domínguez-Rodríguez et al., 2011; Román, Prieto, y Mancilla, 2004; RosalesCastillo, Torres-Meza, Olaiz-Fernández, y Borja-Aburto, 2001; Valenciana, 1999)

Para que la contaminación del aire produzca efectos sobre la salud no se requiere que los niveles de estos sean elevados, incluso cuando están en los parámetros permitidos por la normatividad ocasionan efectos sobre la salud ya que el estar expuesto a dosis bajas durante tiempos prolongados ocasiona estos efectos (Rosales-Castillo et al., 2001).

La calidad del aire en exteriores se contamina por $\mathrm{PM}^{10}, \mathrm{SO}_{2}, \mathrm{NO}_{2}, \mathrm{CO}$ y $\mathrm{O}_{3}$ generados en procesos de combustión de la actividad industrial, de transporte y se reflejan en el perjuicio a los seres vivos (García G et al., 2013; Gavidia et al., 2009; Jaramillo, González, Núñez, y Portilla, 2013; Lacasaña-Navarro, AguilarGarduño, y Romieu, 1999), la temperatura y la humedad propician el contagio de infecciones respiratorias (De La Rosa et al., 2002; Prieto Gómez et al., 2011).

La calidad del aire de interiores como las viviendas, el trabajo o sitios cerrados de permanencia representa riesgo para la población, cuando está deteriorado por la contaminación química, humedad, temperatura, o microorganismos (Aguinaga y Lazo, 2003; De La Rosa et al., 2002; Finkelman et al., 1994), las personas pasan la mayor parte del tiempo en el ambiente interior (Carazo Fernández, Fernández Álvarez, González-Barcala, y Rodríguez Portal, 2013; Rivas R et al., 2008), tienen deficiente ventilación comparado con los ambientes exteriores (Cáceres et al., 2001) que aumentan la concentración de partículas.

El ambiente interior se contamina por combustión de leña (A. Cortés y Ridley, 2013) o derivados del petróleo, humo de cigarrillo (Chang y Gershwin, 2004; Gavidia et al., 2009; Prieto Gómez et al., 2011), condiciones de paredes, pisos y techos de las viviendas, procesos industriales, y el uso de sustancias químicas.

Los efectos de contaminantes en el aire pueden ser agudos causando irritación de ojos y nariz, aumento de las Infecciones Respiratorias Agudas, Infección Respiratoria Aguda Grave, cuadros clínicos de asma, efectos crónicos como la enfermedad pulmonar obstructiva crónica y cáncer pulmonar y la respectiva mortalidad por cada una de estas morbilidades (Arci- 
niegas, Rodríguez, Pachón, Sarmiento, y Hernández, 2016; Calderón, Recinos, Fernández, y Roca, 2012; Feo Brito y Mur Gimeno, 2011; Flores B, Solís S, Fortt Z, y Valdivia C, 2010; Franco y Christian, 2012; García, Toro, y Gómez, 2013; Herrera, 2013; Mezquía Valera, Cumba Abreu, Aguilar Valdés, García Roche, y Acosta Quintana, 2011; Prieto C et al., 2007; Torres, Londoño, Vasco, y Molina, 2011).

El indicador sintético de calidad del aire evidencia la presencia de casos afecciones del sistema respiratorio (infección respiratoria aguda, infección respiratoria aguda grave, e infecciones similares a la influenza), presentes en todos los municipios del Meta, evidencian que la calidad del aire está comportándose como un factor de riesgo.

Los resultados obtenidos en la construcción del indicador sintético para el factor ambiental en la calidad del aire evidencian grados de afectación diferentes entre los municipios, por ejemplo: Puerto Gaitán obtuvo 5210 puntos comparado con Vistahermosa que obtuvo 414 puntos en la escala del indicador, lo anterior evidencia diferencias en los gradientes de exposición a las variables ambientales ligadas a la calidad del aire y el clima entre los municipios.

La fauna como determinante en la salud: La fauna comparte su habitad con los seres humanos, aunque el hombre transforma las características ambientales en sus procesos urbanísticos e invade el habitad natural con el aumento de sus fronteras de desarrollo (Ramos, 2010) y afecta el desarrollo natural de la fauna, esta se adapta y convive en medio de los asentamientos humanos, conformando ecosistemas de especies no humanos (Alcalá, Soto, Lebgue, y Sosa, 2007).

La salud humana es afectada por la presencia de fauna, ya que esta es huésped de microorganismos patógenos que afectan el estado de salud (Ortega-Pacheco, 2001; Ramos, 2010), el proceso de relación entre fauna y humanos ocurre en condiciones ambientales naturales o condiciones en ambientales antrópicas, dichas condiciones determinan la presencia de fauna (García, 2013) causante de morbimortalidad humana, por esto la morbimortalidad asociada a la fauna podría ser usada como indicador del estado y características ambientales (Adolfo Gerardo et al., 2007; Beldoménico, 2008).

La fauna que en su interacción con humanos impacta la salud de manera directa o sirviendo de vector para organismos patógenos (García, 2013), por esta razón las enfermedades ocasionas por la fauna han sido clasificadas en dos grandes grupos: en enferme- dades trasmitidas por vectores (ETV) y en enfermedades zoonótica (Cruz-Reyes, 2009).

La presencia de animales domésticos y silvestres, en asentamientos humanos o cerca de estos asentamiento determina la aparición de zoonosis (Anaya y Lepe, 2009), la enfermedades zoonótica son infecciones o infestaciones en la cuales participa en su cadena de transmisión a humanos los animales vertebrados (Chiodo y Basualdo, 2011; Ledesma, 2010; Pradilla, Mantilla, y Badillo, 2009; Rivas et al., 2012; Seleem, Boyle, y Sriranganathan, 2010; Venza y Nava, 2011), alguna zoonosis han tenido repercusiones mundiales mediante pandemias (Morse et al., 2012) bien sea por contacto directo o indirecto (Cruz-Reyes, 2009).

Las enfermedades trasmitidas por vectores ocurren por la interacción natural de insectos con los seres humanos, estos sirven como medio de transmisión de microorganismos que causan morbilidades y mortalidad (Cerda, Valdivia, Valenzuela, Teresa, y Venegas, 2008; L. A. Cortés y Fernández, 2008; Flores B et al., 2010; Mattar y Arrieta, 2012; Olano, Carrasquilla, y Méndez, 1997; Ríos, Arboleda, Montoya, Alarcón, y Parra-Henao, 2011), la convivencia entre insectos y seres humanos esta mediada por factores ambientales como: humedad, temperatura, fuentes hídricas, cercanía al habitad natural, hacinamiento, altitud, el ordenamiento territorial, las características de la vivienda, la calidad y acceso al recurso hídrico, el aprovisionamiento de recurso hídrico en el interior de la viviendas (Guzmán, 2002) etc.

Los municipios del departamento del Meta como cualquier otro desarrollo urbano poseen área urbana y rular desarrolladas por sus habitantes (Ramos, 2010), estas áreas son un ecosistema que incluye especies no humanas (Adolfo Gerardo et al., 2007; Alcalá et al., 2007; Vásquez-Trujillo et al., 2008), y zona rural donde habitan fauna silvestre (Beldoménico, 2008), las cuales viven en relación ecosistémica con los pobladores de los municipios y en esta interacción la fauna participa en el proceso de enfermedad (Adolfo Gerardo et al., 2007), en los municipios del departamento del Meta la morbilidad y la mortalidad relacionada con la fauna que se encontró en este estudio fue: Malaria, Dengue, Leishmaniasis. Chagas, Accidente Ofídico, Fiebre Amarilla, Agresión animal, la fauna, bien sea como vector, como reservorio, o como hospedero en la historia natural de la enfermedad.

Los resultados obtenidos en la construcción del indicador sintético para el factor ambiental faunístico evidencian grados de afectación diferentes entre los municipios, por ejemplo: Vistahermosa obtuvo 2139 
puntos comparado con San Juanito que obtuvo 77 puntos en la escala del indicador, lo anterior evidencia diferencias en los gradientes de exposición a las variables ambientales ligadas a la fauna entre los municipios.

Las sustancias químicas como determinante de la salud: La exposición humana a sustancias químicas ocasiona efectos sobre la salud (Vargas, 2005) y dependiendo de la concentración de la sustancia en el ambiente y el tiempo de exposición, varia el nivel del impacto, además la exposición varia de concentración en ambiente exterior o interior (Vargas y Pulgarín, 2005).

El desarrollo humano ha generado diversidad de productos químicos para su uso en actividades antrópicas, en Colombia se conocen entre 80.000 a 100.000 sustancias químicas (Gutiérrez Lesmes y Martinez Agudelo, 2016) de uso común la cuales afectan la salud (Uribe et al., 2012), diversas sustancias químicas circulan en los municipios de Meta, ocasionando morbimortalidad relacionada con ellas (Cárdenas, Silva, Morales, y Ortiz, 2005; Montaño, Gómez, Palacio, y Galvis, 2013), en los años analizados en la presente investigación, se registraron casos de Intoxicación por plaguicidas, Intoxicación metales pesados, Intoxicación por solventes, Intoxicación sustancias otras químicas siendo la intoxicación más común la ocasionada por plaguicidas con el $73,8 \%$ de los casos, seguida de la intoxicación con otras por otras sustancias con el 23,35 de los casos.

El Meta es un departamento caracterizado por alta producción agrícola (Mincomercio, 2013), esto explicaría la ocurrencia mayoritaria de morbimortalidad atribuible a plaguicidas (Gutierrez Lesmes, Martinez Agudelo, y Rodriguez Loboa, 2016), los cuales son usados para el control biológico, exponiendo la población a agentes tóxicos, ocasionando enfermedad y muerte por intoxicación (González Vides, 2011; Tabares, Juan, López, y Yolanda, 2011; Zamora, Torres, Rodríguez, y Zamora, 2011).

Las exposiciones pueden estar ocurriendo de forma accidental o por exposición ambiental, los plaguicidas usados en aspersión sobre cultivos producen contaminación del aire (García-Gutiérrez y Rodríguez-Meza, 2012), el agua (Lans, Marrugo, y Díaz, 2008; TobónMarulanda, López-Giraldo, y Paniagua-Suárez, 2010), y los alimentos (Murcia y Stashenko, 2008; M. Pérez et al., 2009), sumado a esto ocurre exposición ocupacional (Gómez-Pérez et al., 2011; Larrea Poma, Tirado Bustillos, y Ascarrunz, 2010; Yucra, Gasco, Rubio, y González, 2008) y el libre acceso a estas sustancias permite que sean utilizadas intentos de suicidio (Gon- zález, 2011; S. Pérez, Álvarez, Baldo, y Capote, 2012) o que ocurran incidentes por consumo accidental (Instituto Nacional de Salud, 2014).

Los resultados obtenidos en la construcción del indicador sintético para el factor sustancias químicas evidencian grados de afectación diferentes entre los municipios por ejemplo: El dorado obtuvo 410 puntos comparado con Puerto Concordia que obtuvo 1,5 puntos en la escala del indicador, lo anterior evidencia diferencias en los gradientes de exposición a las variables ambientales ligadas a las sustancias químicas (Gutiérrez Lesmes, Loboa Rodríguez, y Plata Casas, 2017).

\section{Conclusiones}

Existen efectos en los municipios del departamento de Meta relacionados con factores ambientales como: sustancia químicas, agua, aire y fauna, la evidencia sobre la existencia de un efecto causado por estos factores se deriva de la presencia de morbilidad y mortalidad en diferentes eventos en salud bajo vigilancia epidemiológica como: Intoxicación por Plaguicidas, Intoxicación Metales Pesados, Intoxicación por Solventes, Intoxicación por Sustancias Químicas, Accidente Ofídico, Agresión por animales potencialmente transmisores de Rabia, Enfermedad Diarreica Aguda, Hepatitis A, dengue, Malaria, Leishmaniasis, Chagas, y enfermedad similar a influenza

Este indicador es una herramienta adecuada al permitir acumular los efectos en una misma escala de medición y realizar una caracterización de los factores ambientales presentes en los municipios y evidenciar diferencias en el gradiente de exposición basado en los efectos sobre los perfile epidemiológicos de los municipios.

Es una herramienta económica al usar el sistema de información de vigilancia en salud pública y la red hospitalaria existente.

\section{Recomendaciones}

Es indispensable iniciar procesos con enfoque en salud ambiental en los factores hallados como precursores de efectos sobre los perfiles epidemiológicos en los municipios del Meta.

La academia, los entes territoriales, las autoridades ambientales y de salud del Meta deben iniciar el reconocimiento del ambiente como determinante en la salud de sus habitantes, haciéndose participe en las decisiones que afectan la calidad ambiental en la que viven su población 
Los resultados de la presente investigación evidencian la necesidad de construir un diagnóstico ambiental departamental que permita medir y evaluar los factores ambientales hallados: la calidad y acceso al agua, calidad el aire (contaminación y clima), la interacción del hombre con la fauna (ecosistemas) y el uso de sustancia químicas incluyendo sus características, niveles, fuentes y usos.

Los indicadores generados en esta investigación pueden ser consultados como diagnóstico de la salud ambiental de los municipios del Meta, y ser considerados como la línea base para la construcción de una línea de investigación.

\section{Potencial conflicto de intereses}

En el presente artículo no hay conflicto de intereses.

\section{Fuentes de financiación}

Universidad de los Llanos.

\section{Referencias}

Álvarez-Pérez AG, García-Fariñas A, Bonet-Gorbea M. Pautas conceptuales y metodológicas para explicar los determinantes de los niveles de salud en Cuba. Rev Cuba Salud Pública. 2007;33(2):1-16.

Aguinaga M, Lazo G. Relación entre variables climáticas y casos de Infección Respiratoria Aguda en la Provincia de Callao 2001. Rev Peru Epidemiol. 2003;11(1):1-18.

Alcalá J, Soto R, Lebgue T, Sosa M. Percepción comunitaria de la flora y fauna urbana en la ciudad de Chihuahua, México. Revista Latinoamericana de Recursos Naturales. 2007;3(1): 58-64.

Álvarez-Miño L, Cantillo-Matos K, Rico Gutiérrez K, Salazar A. Acceso y calidad del agua para el consumo humano en Santa Marta como indicador de inequidad en salud. Univ Salud. 2013;15:113-122.

Álvarez-Sintes R, Álvarez-Castro MR, Toledo-Vila H. Asociación entre manifestaciones respiratorias atópicas y contaminantes primarios de la atmósfera. Rev Cuba Med Gen Integral. 1997;13:212-221.

Anaya J, Lepe S. Conocimientos sobre higiene en el manejo de alimentos en hogares y comercios de seis localidades rurales de Gómez Farias. Jalisco. Rev Med UV. 2009;9:30-34.

Hernández-Antonio A, Hasen AM. Uso de plaguicidas en dos zonas agrícolas de México y evaluación de la contaminación de agua y sedimentos. Rev Int Contam Ambient. 2011;27(2): 115-127.

Arciniegas A, Rodríguez C, Pachón J, Sarmiento H, Hernández LJ. Estudio de la morbilidad en niños menores a cinco años por enfermedad respiratoria aguda y su relación con la concentración de partículas en una zona industrial de la ciudad de Bogotá Acta Nova. 2016;3(2):145-154.
Puente-Burgos A, García-Rodríguez J, Polo-Alvarado B, Garcia C. 2008. Evaluación ambiental estratégica para la formulación de política en materia de salud ambiental para Colombia con énfasis en contaminación atmosférica en centros urbanos informe ejecutivo final. Universidad Javeriana. Pp. 1-132.

Barrios-Casas S, Peña-Cortés F, Osses-Bustingorry S. Efectos de la contaminación atmosférica por material particulado en las enfermedades respiratorias agudas en menores de 5 años. Ciencia y enfermería. 2004;10:21-29.

Beldoménico P. Enfermedad y fauna: foco de creciente interés para las ciencias biomédicas. APRONA Bol Cien. 2008;40:46-55.

Cáceres D, Adonis M, Retamal C, Ancic P, Valencia M, Ramos X, Gil L. Contaminación intradomiciliaria en un sector de extrema pobreza de la comuna de La Pintana. Rev Med Chile. 2001;129(1):33-42.

Calderón EC, Recinos ALD, Fernández AJA, Roca GC. Asma y broncoespasmo inducido por el ejercicio. Canarias Pediátrica. 2012;38(3):132-137.

Calleros E, Alarcón M, Pérez R, Cueto J, Moran J, Sanín L. Evaluación de riesgo sistémico y niveles de metahemoglobina en niños que consumen agua contaminada por nitratos. Ingeniería. 2012;16(3):183-194.

Carazo-Fernández L, Fernández-Álvarez R, González-Barcala FJ, Rodríguez-Portal JA. Contaminación del aire interior y su impacto en la patología respiratoria. Arch Bronconeumol. 2013;49(1):22-27.

Cárdenas O, Silva E, Morales L, Ortiz J. Estudio epidemiológico de exposición a plaguicidas organofosforados y carbamatos en siete departamentos colombianos, 1998-2001. Biomédica. 2005;25:170-180.

Castro P, Vera J, Cifuentes L, Wellenius G, Verdejo H, Sepúlveda L, Llevaneras S. Polución por material particulado fino (PM 2,5) incrementa las hospitalizaciones por insuficiencia cardiaca. Rev chil Cardiol. 2010;29:306-314.

Cerda J, Valdivia G, Valenzuela B, Teresa M, Venegas J. Cambio climático y enfermedades infecciosas: un nuevo escenario epidemiológico. Rev Chil Infectol. 2008;25(6):447-452.

Córdoba MA, Del Coco VF, Basualdo JA. Agua y salud humana. Química Viva. 2010;9(3):105-119.

Correa-Restrepo F, de la Ossa Arteaga A, Vallejo-Chanci Z. Regulación ambiental en Colombia: el caso de la tasa retributiva para el control de la contaminación hídrica. Semeste Económico. 2011;10(19):29-46.

Cortés A, Ridley I. Efectos de la combustión a leña en la calidad del aire intradomiciliario: La ciudad de Temuco como caso de estudio. Revista INVI. 2013;28(78):257-271.

Cortés LA, Fernández JJ. Especies de Lutzomyia en un foco urbano de leishmaniasis visceral y cutánea en El Carmen de Bolívar, Bolívar, Colombia. Biomédica. 2008;28(3):433-440.

Cruz-Reyes A. 2009. Fauna feral, fauna nociva y zoonosls. In U. N. A. d. México. (Ed.), Biodiversidad del ecosistema del Pedregal de San Ángel. 
Chang C, Gershwin ME. Indoor air quality and human health. Clinical reviews in allergy \& immunology. 2004;27(3):219-239.

Chiodo P, Basualdo JA. 2011. Temas de Zoonosis IV. Toxocariosis. Veterinaria. 2011;28(280):1852-1317X.

de Jesús Alba J, Ortega JL, Álvarez G, Cervantes M, Ruiz E, Urtiz N, Martínez A. Riesgos microbiológicos en agua de bebida: una revisión clínica. Química Viva. 2013;12(3):215-233.

De La Rosa Md C, Mosso Md IA, Ullán C. El aire: hábitat y medio de transmisión de microorganismos. Observatorio medioambiental. 2002;5:375-402.

Domínguez-Rodríguez A, Abreu-Afonso J, Rodríguez S, Juárez-Prera RA, Arroyo-Ucar E, Jiménez-Sosa A, Avanzas P. Estudio comparativo de las partículas en aire ambiente en pacientes ingresados por insuficiencia cardiaca y síndrome coronario agudo. Revista Española de Cardiología. 2011;64(8):661-666.

Escobedo RC, Hoyos MC, Zavalaga FL. Factores asociados a malaria en el Distrito de Llochegua, Valle de los Ríos Apurimac y Ene, Provincia de La Mar, Ayacucho-Perú-2003. Rev Medica Hered. 2012;20(3):139.

Espinoza BD, Segura CM, Rodríguez FA. Implicaciones en la salud humana de la gastroenteritis aguda por norovirus como producto de la contaminación hídrica. Tecnología en Marcha. 201124(4):3.

Esquicha JA, Falcón N, Oshiro S. Características clínicas y epidemiológicas de los pacientes con neurocisticercosis en un hospital general de Lima. Rev Medica Hered. 2012;23:4-10.

Feo Brito F, Mur Gimeno P. Asma y contaminación. ESPACIO ASMA. 2011;4(1):8.

Finkelman J, Corey G, Calderon RL. 1994. Epidemiología ambiental: un proyecto para América Latina y el Caribe: Centro Panamericano de Ecología Humana y Salud.

Flores BC, Solís SMT, Fortt ZA, Valdivia CG. Sintomatología respiratoria y enfermedad pulmonar obstructiva crónica y su asociación a contaminación intradomiciliaria en el Área Metropolitana de Santiago: Estudio Platino. Rev Chil Enferm Respir. 2010;26:72-80.

Franco A, Christian B. 2012. Prevalencia de asma y su relación con la contaminación del medio externo en los niños y niñas de 2 a 5 años en los centros de desarrollo infantil municipales de la ciudad de Cuenca, 2012.

García-Gutiérrez C, Rodríguez-Meza GD. Problemática y riesgo ambiental por el uso de plaguicidas en Sinaloa. Ra Ximhai. 2012;8(3):.

García CEO, Toro MV, Gómez JC. EPOC, bronquitis crónica y síntomas respiratorios, asociados a la contaminación por pm10 en la ciudad de Medellín (Colombia). Revista Med. 2013;21(1):21-28.

García GM, Ramírez SH, Ulloa GH, García GO, Meulenert PÁ, Alcalá G). Concentración de contaminantes $\mathrm{SO} 2, \mathrm{NO} 2$ y correlación con $\mathrm{H}+$, SO4-2 y NO3- durante la temporada de lluvias en la Zona Metropolitana de Guadalajara, Jalisco, México. Rev Chil Enferm Respir. 2013;29:81-88.

García M. 2013. Fauna Nociva como Factor de Riesgo de Enfermedades Zoonóticas. Universidad técnica de Machala Machala.
Gavidia T, Pronczuk J, Sly PD. Impactos ambientales sobre la salud respiratoria de los niños: Carga global de las enfermedades respiratorias pediátricas ligada al ambiente. Rev Chil Enferm Respir. 2009;25:99-108.

Gómez-Pérez R, Rojas G, Miranda-Contreras L, Cruz I, Berrueta L, Salmen S, Colmenares M. Efectos de exposición ocupacional a plaguicidas sobre la integridad de la cromatina espermática. Rev Venez Endocrinol Metab. 2011;9(2):67-78.

González G. 2011. Intoxicacion por plaguicidas: casuística del Hospital Universitario del Caribe y de la Clínica Universitaria San Juan de Dios de Cartagena. 2009-2010. Universidad Nacional de Colombia.

González Vides G. 2011. Intoxicacion por plaguicidas: casuística del Hospital Universitario del Caribe y de la Clínica Universitaria San Juan de Dios de Cartagena. 2009-2010/Pesticide poisoning: University Hospital cases of the Caribbean and the University Clinic of San Juan de Dios Cartagena. 2009 to 2010. Universidad Nacional de Colombia.

Guillen A, González M, Gallego L, Suárez B, Heredia HL, Hernández $\mathrm{T}$, Salazar J. Presencia de protozoarios intestinales en agua de consumo en la comunidad 18 de Mayo. Estado Aragua-Venezuela, 2011. B Malariol Salud Amb. 2013;53(1):29-36.

Gutiérrez-Lesmes O, Martinez-Agudelo C, Rodriguez-Loboa N. Situación epidemiológica de la intoxicación con plaguicidas en los municipios del Meta, periodo 2009-2014. Salud, Historia y Sanidad On-Line. 2016;11(1):27-35.

Gutiérrez-Lesmes OA, Loboa-Rodríguez NJ, Plata-Casas LI. Situación epidemiológica de la intoxicación por sustancias químicas en el departamento del Meta-Colombia, periodo 2009-2014. Biosalud. 2017;16(1):30-42.

Gutiérrez-Lesmes OA, Martinez-Agudelo C. Indicador sintético para la medición de la necesidad de Investigación y gestión ambiental basado en morbimortalidad Ocurrida en 2009-2012, Meta, Colombia. Luna Azul. 2016;42:154-166. doi: 10.17151/ luaz.206.42.10

Guzmán M. El dengue y el dengue hemorrágico i una entidad olvidada. Rev Cubana Med Trop. 2002:54(3), 169-170.

Hernández-Flórez LJ, Aristizabal-Duque G, Quiroz L, Medina K, Rodríguez-Moreno N, Sarmiento R, Osorio-García SD. Contaminación del aire y enfermedad respiratoria en menores de cinco años de Bogotá, 2007. Rev Salud Pública. 2013;15(4):552-565.

Herrera AE. 2013. Factores de riesgo correlacionados a las infecciones respiratorias agudas en niños menores de 5 años de edad atendidos en el subcentro de salud 18 de octubre de la ciudad de Machala, durante julio 2011-marzo 2012

Instituto Nacional de Salud. 2014. Protocolo de vigilancia en salud publica intoxicaciones por sustancias quimicas.

Jacinto E, Aponte E, Arrunátegui-Correa V. Prevalencia de parásitos intestinales en niños de diferentes niveles de educación del distrito de San Marcos, Ancash, Perú. Rev Medica Hered. 2012;23:235-239.

Janet VAL, Fernanda EL, Parra HLG. Identificación de fuentes terrestres de contaminación y cálculo de las cargas de contaminantes 
en el área de influencia de la ciénaga grande de santa marta, Caribe Colombiano. Bol Invest Mar Cost. 2013;42:1.

Jaramillo M, González DE, Núñez ME, Portilla G. Índice integrado de calidad del aire para ciudades colombianas. Rev Fac Ing Univ Antioquia. 2009 2013;(48):97-106.

Junkin M, Eugene F. Agua y salud humana: Editorial Limusa, S.A de $\mathrm{CV}$.

Lacasaña-Navarro, M., Aguilar-Garduño, C., \& Romieu, I. (1999). Evolución de la contaminación del aire e impacto de los programas de control en tres megaciudades de América Latina. Salud Pública de Mex. 1988;41(3):203-215.

Lans E, Marrugo JL, Díaz B. Estudio de la contaminación por pesticidas organoclorados en aguas de la Ciénaga Grande del Valle Bajo del río Sinú. Temas Agrarios. 2008;13(1):49-56.

Larrea-Poma M, Tirado-Bustillos N, Ascarrunz E. Daño genotóxico por exposición a plaguicidas en agricultores del Municipio de Luribay. Biofarbo. 2010;18:31.

Ledesma V. leptospirosis en el Estado de tabasco. Revista de la Facultad de Medicina de la UNAM. 2010;54:4-9.

Mattar S, Arrieta G. Enfermedades emergentes y reemergentes: importancia de las enfermedades transmitidas por vectores. Infectio, 2012;12(1S)

Medina D, Bevilacqua M, Cárdenas L, Morales LG, Rubio-Palis Y, Martínez A, Magris M. Mapa de riesgo de transmisión de malaria en la cuenca del río Caura, Venezuela Risk map of malaria transmission in the Caura river basin, Venezuela. Bol Malariol Salud Ambient. 2011; 51(2):129-144.

Melgar-Pérez J. 2013. Urgencias hospitalarias pediátricas a lo largo de una década: influencia del calendario, condiciones meteorológicas y contaminación atmosférica.

Mezquía-Valera A, Cumba-Abreu C, Aguilar-Valdés J, García-Roche R, Acosta-Quintana L. Condiciones ambientales riesgosas para las infecciones respiratorias agudas en escolares de primaria. Revista Cubana de Medicina General Integral. 2011;27:155-160.

Mincomercio. (2013). Departamento de Meta. https://www.mincomercio.gov.co/publicaciones.php?id=16724

Decreto 1200 Por el cual se determinan los Instrumentos de Planificación Ambiental y se adoptan otras disposiciones (2004).

Ministerio de la Protección Social. 2012. Gestión para la vigilancia entomológica y control de la transmisión de dengue. Colombia.

Moiso A, Barragán H. 2007. Determinantes de la salud. Fundamentos de la Salud Pública. Argentina, La Plata: Universidad Nacional de La Plata, Editorial EDULP.

Molina-Esquivel E, Meneses-Ruiz E. Evaluación epidemiológica del impacto de los contaminantes del aire: Propuesta metodológica. Revista Cubana de Higiene y Epidemiología. 2003;41:0-0.

Montaño C, Gómez C, Palacio T, Galvis A. 2013. Informe del comportamiento epidemiológico de los eventos de interés en salud pública y de las actividades realizadas en el sistema de vigilancia durante el primer trimestre de 2013 en el Departamento del Meta. from http://saludmeta.gov.co/wp-
content/uploads/2013/04/Informe-comparativo-primer-trimestre-a\%C3\%B1 os-2012-2013.pdf

Morse SS, Mazet JA, Woolhouse M, Parrish CR, Carroll D, Karesh WB, Daszak P. Prediction and prevention of the next pandemic zoonosis. The Lancet. 2012;380(9857):1956-1965.

Murcia A, Stashenko E. Determinación de plaguicidas organofosforados en vegetales producidos en Colombia. Agro sur. 2008.;36(2):71-81.

Olano V, Carrasquilla G, Méndez Fn. Transmisión de la malaria urbana en Buenaventura, Colombia: aspectos entomológicos. PAN AMERICAN JOURNAL OF PUBLIC HEALTH, 1997;1:287-294.

Oller-Arlandis V, Sanz-Valero J. Cáncer por contaminación química del agua de consumo humano en menores de 19 años: una revisión sistemática. Revista Panamericana de Salud Pública. 2012:32:435-443.

Ordóñez G. Salud ambiental: conceptos y actividades. Rev Panam Salud Publica. 2000;7(3): 137-147.

Organización-Panamericana-de-la-Salud. (2001). Informe Regional sobre la Evaluación 2000, en la Región de las Américas: agua potable y saneamiento, estado actual y perspectivas. Retrieved from: http:// www.bvsde.ops-oms.org/bvsaas/e/fulltext/infregio/infregio.pdf.

Ortega-Pacheco A. La sobrepoblación canina: un problema con repercusiones potenciales para la salud humana. Rev Biomed. $2001 ; 12(4): 288-289$

Pérez-Jiménez D, Diago-Garrido Y, Corona-Miranda B, Espinosa-Díaz R, González-Pérez JE. Enfoque actual de la salud ambiental. Revista Cubana de Higiene y Epidemiología. 2011;49(1):84-92.

Pérez M, Segura A, García R, Colinas T, Pérez M, Vázquez A, Navarro $\mathrm{H}$. Residuos de plaguicidas organofosforados en cabezuela de brócoli (Brassica oleracea) determinados por cromatografía de gases. Revista internacional de contaminación ambiental. 2009;25(2):103-110.

Pérez S, Álvarez M, Baldo D, Capote B. Intoxicaciones agudas por plaguicidas consultadas al Centro Nacional de Toxicología durante el bienio 2007-2008. Revista Cubana de Medicina Militar. 2012;41(4):415-422.

Posada M, Carronquino MJ, Soldevilla LdIP. Indicadores de salud ambiental. Revista de Salud Ambiental. 2004;4(1-2):1-7.

Posada M, Carroquino J, Soldevilla L. Indicadores de Salud Ambiental. Revista de Salud Ambiental. 2004;4(1-2):1-7.

Pradilla G, Mantilla JC, Badillo R. Encefalitis rábica humana por mordedura de murciélago en un área urbana de Colombia. Biomédica, 2009;29(2):191-203.

Prieto CMJ, Mancilla FP, Astudillo OP, Reyes PÁ, Román AO. Exceso de morbilidad respiratoria en niños y adultos mayores en una comuna de Santiago con alta contaminación atmosférica por partículas. Revista médica de Chile. 2007;135:221-228.

Prieto-Gómez E, Robaina-Rivero EdR, González-González GA, SotoGarcía E, Fleitas- Echeverría D, García-Marín M. Infecciones respiratorias altas recurrentes en niños menores de 5 años. Jagüey Grande. 2008-2009. Revista Médica Electrónica. 2011;33:401407. 
Prüss-Üstün, Annette-Corvalán C. 2006. Ambientes saludables y prevención de enfermedades: hacia una estimación de la carga de morbilidad atribuible al medio ambiente (OMS Ed. Vol. 1): OMS.

Quezada A. Los objetivos del milenio: algunas tareas para todos. Revista chilena de pediatría. 2006;77(2):123-126.

Ramos JG. Zoonosis. Gaceta Médica de México. 2010;146:436-436.

Ríos JF, Arboleda M, Montoya AN, Alarcón EP, Parra-Henao GJ. Probable brote de transmisión oral de enfermedad de Chagas en Turbo, Antioquia. Biomédica: Revista del Instituto Nacional de Salud. 2011;31(2).

Rivas C, Greif G, Coitinho C, Araújo L, Laserra P, Robello C. Primeros casos de tuberculosis pulmonar por Mycobacterium bovis: una zoonosis reemergente en Uruguay. Revista Médica del Uruguay. 2012;28(3):209-214.

Rivas RE, Barrios CS, Dorner PA, Osorio SX. Fuentes de contaminación intradomiciliaria y enfermedad respiratoria en jardines infantiles y salas cunas de Temuco y Padre Las Casas, Chile. Revista médica de Chile. 2008;136:767-774.

Román O, Prieto MJ, Mancilla P. Contaminación atmosférica y daño cardiovascular. Revista médica de Chile. 2004;132(6):761-767.

Romero-Placeres $M$, Más-Bermejo $P$, Lacasaña-Navarro $M$, RojoSolís MMT, Aguilar-Valdés J, Romieu I. Contaminación atmosférica, asma bronquial e infecciones respiratorias agudas en menores de edad, de La Habana. Salud Pública de México. 2004;46;222-233.

Rosales-Castillo JA, Torres-Meza VM, Olaiz-Fernández G, BorjaAburto $\mathrm{VH}$. Los efectos agudos de la contaminación del aire en la salud de la población: evidencias de estudios epidemiológicos. Salud Pública de México. 2001;43(6):544-555.

Schuschny A, Soto H. 2009. Diseño de indicadores compuestos de desarrollo sostenible.

Seleem MN, Boyle SM, Sriranganathan N. Brucellosis: a re-emerging zoonosis. Veterinary microbiology. 2010;140(3):392-398.

Silva H, Villalobos B, Ortega D. Calidad bacteriológica del agua del río Cataniapo, municipio Atures, estado Amazonas, Venezuela Sanitary quality of the hydrographic basin of the Cataniapo river, Atures municipality, Amazonas state, Venezuela. Bol. malariol. salud ambient. 2012;52(1):99-105.

Tabares L, Juan C, López A, Yolanda L. Salud y riesgos ocupacionales por el manejo de plaguicidas en campesinos agricultores, municipio de Marinilla, Antioquia, 2009. Revista Facultad Nacional de Salud Públic. 2011;29(4).

Tobón-Marulanda FÁ, López-Giraldo LA, Paniagua-Suárez RE. Contaminación del agua por plaguicidas en un área de Antioquia. Revista de Salud Pública. 2010;12(2):300-307.

Tobón M, López L. Genotoxicidad del agua contaminada por plaguicidas en un área de Antioquia. Revista MVZ Córdoba. 2011;16(2):

Torres AMH, Londoño CAE, Vasco GJM, Molina JEO. Patologías respiratorias en niños preescolares y su relación con la concentración de contaminantes en el aire en la ciudad de Me- dellín (Colombia). Revista Ingenierías Universidad de Medellín. 2011;10(19):21-31.

Uribe M, Castro R, Paéz I, Carvajal N, Barbosa E, León L, Díaz S. Impacto en la salud y el medio ambiente por exposición a plaguicidas e implementación de buenas prácticas agrícolas en el cultivo de tomate, Colombia, 2011. Revista Chilena de Salud Pública. 2012;16(2):96-106.

Valderrama JFN, Baena JAP, Pérez FJM. Persistencia de plaguicidas en el ambiente y su ecotoxicidad: Una revisión de los procesos de degradación natural. Gestión y Ambiente. 2012;15(3):27-37.

Valenciana G. Efectos de la contaminación atmosférica sobre la salud: una introducción. Rev Esp Salud Púbhca. 1999;73:109-121.

Valenzuela E, Godoy R, Almonacid L, Barrientos M. Calidad microbiológica del agua de un área agrícola-ganadera del centro sur de Chile y su posible implicancia en la salud humana. Revista chilena de infectología. 2012;29:628-634.

Vargas M. La contaminación ambiental como factor determinante de la salud. Revista Española de Salud Pública. 2005;79:117 127.

Vargas M, Pulgarín I. Calidad ambiental interior: bienestar, confort y salud. Rev Esp Salud Pública. 2005;79(2):243-251.

Vásquez-Trujillo A, Santamaría-Herreño E, González-Reina AE, Buitrago-Álvarez LS, Góngora-Orjuela A, Cabrera-Quintero OL. Lutzomyia antunesi, Probable Vector de Leishmaniasis Cutánea en el Área Rural de Villavicencio. Revista de Salud Pública. 2008;10:625-632.

Venza JM, Nava S. El género Rickettsia como agente de zoonosis en el Cono Sur de Sudamérica. Revista Médica del Uruguay. 2011;27(2):98-106.

Vieira SE, Stein RT, Ferraro AA, Pastro LD, Pedro SSC, Lemos M, Saldiva $\mathrm{PH}$. Los contaminantes atmosféricos urbanos son factores de riesgo significativos para el asma y la neumonía en niños: influencia del lugar de medición de los contaminantes. Archivos de Bronconeumología. 2012;48(11):389-395.

Villar, E. Los Determinantes Sociales de Salud y la lucha por la equidad en Salud: desafíos para el Estado y la sociedad civil. Saúde e Sociedade. 2007;16(3):7-13.

World Health Organization. 2004. De la teoría a la práctica: indicadores de salud ambiental infantil: implementación de una iniciativa lanzada en la Cumbre Mundial sobre el Desarrollo Sostenible (in press).

Yassi A, Kjellstrom T, Dekok T, Guidotti T. 2002. Salud ambiental básica: Programa de las Naciones Unidad para el Medio Ambiente Oficina Regional para America y el Caribe.

Yucra S, Gasco M, Rubio J, Gonzales G. Exposición ocupacional a plomo y pesticidas órganofosforados: efecto sobre la salud reproductiva masculina. Revista Peruana de Medicina Experimental y Salud Publica. 2008;25(4):394-402.

Zamora FR, Torres D, Rodríguez N, Zamora FJ. Impacto del uso de plaguicidas sobre los niveles de colinesterasa total en sangre en productores agrícolas del asentamiento campesino Santa Teresa, Municipio Miranda del estado Falcón, Venezuela. Revista de la Facultad de Agronomía. 2011;35(2):56-61. 